SHORT REPORT

\title{
Thixotropy of levator palpebrae as the cause of lagophthalmos after peripheral facial nerve palsy
}

\author{
M Aramideh, J H T M Koelman, P P Devriese, J D Speelman, B W Ongerboer de Visser
}

J Neurol Neurosurg Psychiatry 2002;72:665-667

Patients with facial nerve palsy are at risk of developing corneal ulceration because of lagophthalmos (incomplete closure of the affected eyelid). Lagophthalmos could result from thixotropy of the levator palpebrae muscle-that is, the formation of tight crossbridges between the actin and myosin filaments of the muscle fibres causing stiffness of the muscle-rather than from paralysis of the orbicularis occuli muscle as previously supposed. This possibility was investigated in 13 patients with a peripheral facial nerve palsy in a prospective open study. The levator muscle of the affected eyelid was stretched by manipulation and downward movement of the passively closed upper eyelid for 15 seconds. The amount of lagophthalmos was measured before and immediately after this manoeuvre. In all patients except one there was a clear reduction in lagophthalmos (mean reduction $72 \%$; range 60-100\%). Thus in this setting the lagophthalmos appears to be caused by thixotropy of the levator palpebrae muscle, which has implications for treatment.

$\mathrm{P}$ eripheral facial nerve palsy is the most commonly encountered cranial nerve disorder, with an annual incidence of 35 per $100000 . .^{1-4}$ It has various causes, and there is an association between Bell's palsy-the "idiopathic" form of facial nerve palsy-and hypertension and diabetes mellitus. ${ }^{1-3}$

The functional morbidity and aesthetic abnormalities associated with facial nerve palsy mean that early diagnosis and rapid treatment are required. Prevention of corneal exposure and ulceration caused by lagophthalmos-that is, the incomplete closure of the affected eyelid-is specially important. Many types of treatment have been developed to prevent or to minimise the complications of lagophthalmos after facial nerve palsy. ${ }^{2-4}$

The orbicularis oculi muscle (the eyelid closer) and the levator palpebrae muscle (the eyelid opener) act antagonistically during various eyelid movements. During a blink or voluntary forceful closure of the eyelids, the levator palpebrae relaxes, releasing the passive, elastic downward forces stored in the eyelid ligaments, while the orbicularis oculi muscle contracts, causing rapid lowering of the eyelid. As soon as the orbicularis oculi activity returns to its quiescent state, the levator palpebrae resumes its tonic activity, raising the eyelid to its previous position. ${ }^{5-7}$

At first sight, it seems logical to assume that lagophthalmos is directly caused by paresis of the orbicularis oculi muscle. However, there are several reasons to believe that this is not the case. First, it is known that gentle closure of the eyelids, as occurs during sleep, is not caused by contraction of the orbicularis oculi, but is brought about solely by inhibition of the levator palpebrae ${ }^{5-7}$; however, in patients with facial nerve palsy the affected eyelid remains open during sleep. Second, during downward saccadic movement of the eyes, there is associated downward motion of the upper eyelids and a smooth pursuing downward movement of the eyelid following downward movement of the eyes. These upper eyelid movements are mainly caused by gradual inhibition of levator palpebrae activity. ${ }^{8}$ However, it is our observation that patients with facial nerve palsy and lagophthalmos seem unable to perform these movements properly on the side of paresis.

In the light of these findings, we hypothesised that the lagophthalmos in such patients might reflect thixotropy of the levator palpebrae muscle. Thixotropy is stiffness of a striated muscle because of the formation of tight crossbridges between the actin and myosin filaments within muscle fibres. This thixotropic response might occur because of the disturbed blinking on the side of the facial nerve paresis, the levator muscle not being stretched sufficiently to detach the crossbridges. We have tested this hypothesis by manually stretching the levator palpebrae muscle and measuring the alteration in lagophthalmos.

\section{METHODS}

In a prospective open study, we examined 13 patients with a facial nerve palsy, nine male and four female, aged betwen 17 and 73 years (mean 38 years). Nine had Bell's palsy and the others had facial nerve palsy from various causes. The time interval between the occurrence of facial nerve palsy and our investigation varied from one day to six months (median 18 days). All patients had lagophthalmos of varying degree. The patients were asked to close their eyelids gently, as though they were attempting to sleep, at which point we measured the width of the palpebral fissure and hence the amount of lagophthalmos.

Our intervention consisted of complete passive closure of the affected eyelid, followed by manual stretching of the upper eyelid, and hence of the levator palpebrae, in a downward direction as far as was possible. The levator was stretched for approximately 15 seconds. The patients were then asked to open their eyelids and close them gently again, at which point the degree of lagophthalmos was remeasured. In each patient, one measurement was made before and one after the eyelid stretch manoeuvre.

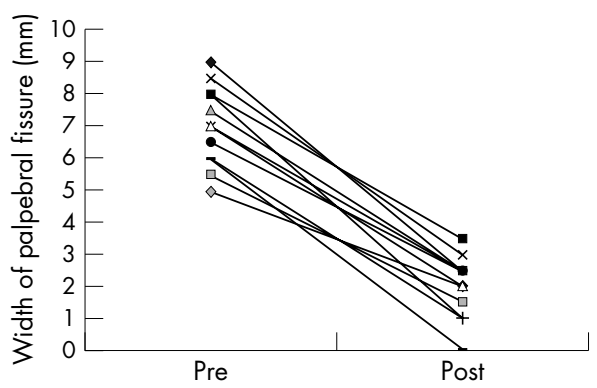

Figure 1 The width of the palpebral fissure measured before and after the upper eyelid stretch manoeuvre in 12 patients with peripheral facial nerve palsy. Each symbol indicates one patient. 

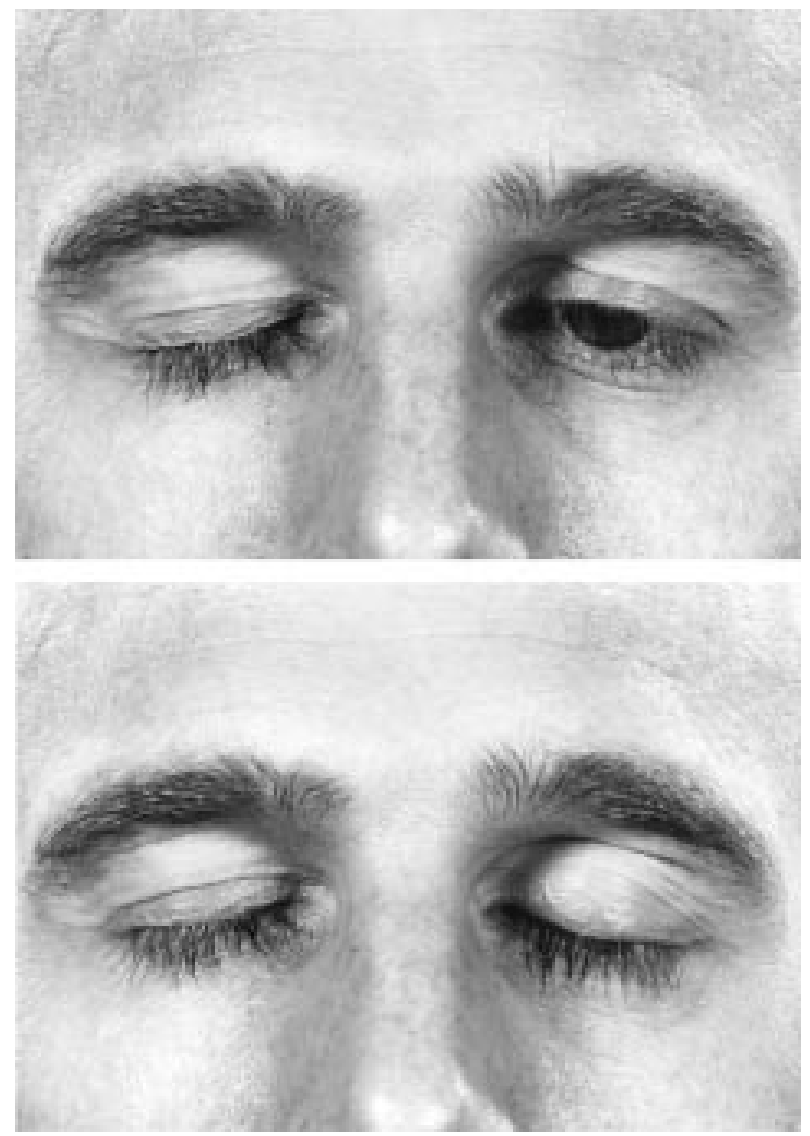

Figure 2 A patient with left sided Bell's palsy. The patient was asked to close the lids gently. The upper photograph shows the amount of lagophthalmos before the lid stretch manoeuvre. The lower photograph shows diminution of lagophthalmos after the manoeuvre.

The patients gave their consent to the procedure but were unaware of any possible effects of the intervention.

\section{RESULTS}

In one patient the affected eyelid could not be stretched properly. In the remaining 12 patients, there was a relevant and significant reduction of lagophthalmos after the intervention. Figure 1 shows the changes in the width of the palpebral fissure measured in these patients. Before the lid stretch manoeuvre, lagophthalmos varied from 9.0 to $5.0 \mathrm{~mm}$ (mean (SD), $7.0(1.2) \mathrm{mm}$ ); after the intervention the range was from 3.5 to $0.0 \mathrm{~mm}$ (mean, $2.0(0.9) \mathrm{mm}$ ). The lagophthalmos was reduced by between $60 \%$ and $100 \%$ (mean $72 \%$ ). Figure 2 shows the amount of lagophthalmos in a patient before and after the lid stretch manoeuvre.

The positive effect of the lid stretch manoeuvre usually diminished within a few minutes, but repeated intervention appeared to increase the duration of the response. All 12 patients noticed an improvement in eyelid closure after our intervention.

\section{DISCUSSION}

In this study, we examined the hypothesis that lagophthalmos following a facial nerve palsy is not directly caused by paresis of the orbicularis oculi but is a result of thixotropy of the levator palpebrae muscle fibres.

The term thixotropy comes from the Greek words "thixis," meaning touch, and "tropos," meaning transformation. ' In striated muscles, thixotropy has been used to describe the dry friction-like behaviour of passive muscle to movement, as distinction from its elastic (length dependent) or viscous (velocity dependent) behaviour. ${ }^{9}$

The stiffness or slackness of a muscle depends on its previous contraction level. The thixotrophic response of muscle fibres results from the formation of stable crossbridges between the actin and myosin filaments, the contractile elements within a muscle fibre. If a muscle remains passive and its length is unchanged, these stable crossbridges stay intact for long periods. A consequence of the crossbridge formation is stiffening of the muscle fibres, as implied by the term thixotropy. ${ }^{10}$ Stretching the muscle detaches the crossbridges.

Under physiological conditions, during every blink the levator palpebrae muscle relaxes and the orbicularis oculi muscle contracts, and this causes the levator to be stretched and the crossbridges to be detached. This physiological mechanism is disturbed in patients with facial nerve palsy. Because of disturbed blinking on the side of the paresis, the levator palpebrae muscle is not stretched sufficiently and so the levator crossbridges remain intact. This will cause excessive stiffness of the levator muscle.

In a group of patients with a facial nerve palsy and lagophthalmos, we manually stretched the levator palpebrae muscle, thereby detaching the crossbridges within the muscle fibres. The effect of this stretch manoeuvre was clinically significant, as the degree of reduction in lagophthalmos achieved would be sufficient to prevent corneal ulceration.

The upper eyelid stretch manoeuvre was initially applied to investigate our hypothesis of the cause of lagophthalmos. The results appear to confirm our explanation of the pathophysiology of this condition. Additional investigations are now required to develop strategies for preventing or treating lagophthalmos. For example, it needs to be shown whether early intervention by stretching the affected eyelid could prevent the occurrence of lagophthalmos or synkinesia. Until such studies are undertaken, we are instructing our patients to close the affected eyelid passively and to stretch the levator muscle manually at repeated intervals through the day.

\section{ACKNOWLEDGEMENT}

We thank Professor M Vermeulen and Dr M Raasveld for their helpful comments during the preparation of the manuscript.

\section{Authors' affiliations}

M Aramideh, J D Speelman, Department of Neurology, Academic Medical Centre, Amsterdam, Netherlands

J H T M Koelman, B W Ongerboer de Visser, Clinical

Neurophysiology Unit, Academic Medical Centre

P P Devriese, Department of Otolaryngology, Facial Research Unit, Academic Medical Centre

Correspondence to: Dr M Aramideh, Department of Neurology, Alkmaar Medical Centre, PO Box 501, 1800 AM Alkmaar, Netherlands;

m.aramideh@mca.nl

Received 19 September 2001

In revised form 8 January 2002

Accepted 18 January 2002

\section{REFERENCES}

1 Katusic SK, Beard CM, Wiederholt WC, et al. Incidence, clinical features and prognosis in Bell's palsy, Rochester, Minnesota, 1968-1982. Ann Neurol 1986;20:622-7.

2 Devriese PP, Schumacher T, Scheide A, et al. Incidence, prognosis and recovery of Bell's palsy. A survey of about 1000 patients. Clin Otolaryngol 1990;15:15-27.

3 Adour KK, Byl FM, Hilsinger RL, et al. The true nature of Bell's palsy: analysis of 1000 consecutive patients. Laryngoscope 1978;88:787801

4 Adour KK. Current concepts in neurology: diagnosis and management of facial paralysis. N Engl J Med 1982;307:348-51. 
5 Evinger C, Manning KA, Sibony PA. Eyelid movements. Mechanisms and normal data. Invest Ophthalmol Vis Sci 1991;32:387-400.

6 Aramideh M, Ongerboer de Visser BW, Devriese PP, et al. Electromyographic features of levator palpebrae superioris and orbicularis oculi muscles in blepharospasm. Brain 1994;117:27-38.

7 Aramideh M, Ongerboer de Visser BW, Koelman JHTM, et al. Motor persistence of orbicularis oculi muscle in eyelid opening disorders. Neurology 1995;45:897-902.
8 Becker W, Fuchs AF. Lid-eye coordination during vertical gaze changes in man and monkey. J Neurophysiol 1988;60:1227-52.

9 Proske U, Morgan DL, Gregory JE. Thixotropy in skeletal muscle and in muscle spindles: a review. Prog Neurobiol 1993:41:705-21.

10 Hagbarth KE, Nordin M, Bongiovanni LG. After-effects on stiffness and stretch reflexes of human finger flexor muscles attributed to muscle thixotropy. J Physiol (Lond) 1995;482:215-23.

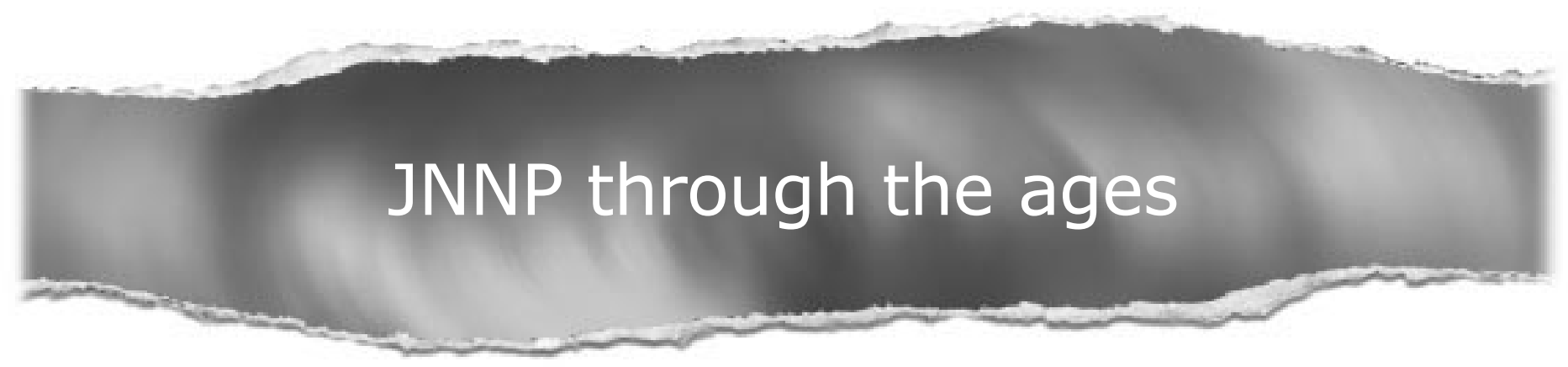

\section{Browse the Archive}

Journal of Neurology, Neurosurgery, and Psychiatry online has an archive of content dating back to 1966.

Full text from 1997; abstracts from 1975; table of contents from 1966

www.jnnp.com 